Journal of

\title{
Implementing a patient-initiated review system for people with rheumatoid arthritis: a prospective, comparative service evaluation
}

\begin{tabular}{|c|c|}
\hline Journal: & Journal of Evaluation in Clinical Practice \\
\hline Manuscript ID & JECP-2015-0365.R1 \\
\hline Wiley - Manuscript type: & Original Article \\
\hline Date Submitted by the Author: & $\mathrm{n} / \mathrm{a}$ \\
\hline Complete List of Authors: & $\begin{array}{l}\text { Goodwin, Victoria; University of Exeter, PenCLAHRC } \\
\text { Paudyal, Priyamvada; Brighton and Sussex Medical School, } \\
\text { Perry, Mark; Plymouth Hospitals NHS Trust, Rheumatology } \\
\text { Day, Nikki; Plymouth Hospitals NHS Trust, Rheumatology } \\
\text { Hawton, Annie; University of Exeter, Medical School } \\
\text { Gericke, Christian; Wesley Research Institute, } \\
\text { Ukoumunne, Obioha; University of Exeter, PenCLAHRC } \\
\text { Byng, Richard; Plymouth University, PenCLAHRC }\end{array}$ \\
\hline Keywords: & health services research, patient-centered care \\
\hline Abstract: & $\begin{array}{l}\text { Background } \\
\text { The management of rheumatoid arthritis (RA) usually entails regular } \\
\text { hospital reviews with a specialist often when the patient is well rather than } \\
\text { during a period of exacerbation. An alternative approach where patients } \\
\text { initiate appointments when they need them can improve patient } \\
\text { satisfaction and resource use whilst being safe. This service evaluation } \\
\text { reports a system wide implementation of a patient-initiated review } \\
\text { appointment system called Direct Access (DA) for people with RA. The aim } \\
\text { was to establish the impact on patient satisfaction of the new system } \\
\text { versus usual care as well as evaluate the implementation processes. } \\
\text { Method } \\
\text { As all patients could not start on the new system at once, in order to } \\
\text { manage the implementation, patients were randomly allocated to DA or to } \\
\text { usual care. Instead of regular follow-up appointments, DA comprised an } \\
\text { education session and access to a nurse-led telephone advice line where } \\
\text { appointments could be accessed within two weeks. Usual care comprised } \\
\text { routine follow-ups with the specialist. Data were collected on patient } \\
\text { satisfaction, service use and outcomes of any contact to the advice line. } \\
\text { Results } \\
\text { Three hundred and eleven patients with RA were assessed as being } \\
\text { suitable for DA. In terms of patient satisfaction, between-group differences } \\
\text { were found in favour of DA for accessibility and convenience, ease of } \\
\text { contacting the nurse and overall satisfaction with the service. Self-reported } \\
\text { visits to the GP were also significantly lower. DA resulted in a greater } \\
\text { number of telephone contacts (incidence rate ratio = } 1.69 ; 95 \% \text { CI } 1.07 \text { to } \\
2.68 \text { ). Hospital costs of the two different service models were similar. Mean }\end{array}$ \\
\hline
\end{tabular}


waiting time for an appointment was 10.8 days

Conclusion

This service evaluation found that DA could be implemented and demonstrated patient benefit in a real world setting. Further research establishing the broader cost-consequences across the whole patient pathway would add to our findings.

\section{SCHOLARONE ${ }^{m}$ \\ Manuscripts}


Implementation of patient-initiated reviews

Implementing a patient-initiated review system for people with rheumatoid arthritis: a prospective, comparative service evaluation

*Victoria A Goodwin, Senior Research Fellow, PhD, University of Exeter Medical School, Room 2.26 South Cloisters, St Luke's Campus, Magdalen Road, Exeter EX1 2LU, Telephone +44 (0) 1392722745

Priyamvada Paudyal, Senior Research Fellow, PhD, Brighton and Sussex Medical School, BN1 9PH

Mark G Perry, Consultant Rheumatologist, MD, Plymouth Hospitals NHS Trust, PL6 8DH

Nikki Day, Lead Rheumatology Nurse, SRN, Plymouth Hospitals NHS Trust, PL6 8DH

Annie Hawton, Senior Research Fellow, PhD, University of Exeter Medical School, EX1 2LU

Christian Gericke, Chief Executive Officer, MD, Wesley Research Institute, Brisbane, QLD 4066

Obioha C Ukoumunne, Associate Professor in Medical Statistics, PhD, University of Exeter Medical School, EX1 2LU

Richard Byng, Professor of Primary Care Research, PhD, Plymouth University Peninsula Schools of Medicine and Dentistry, PL4 8AA

*corresponding author

Key words: person-centred care, rheumatoid arthritis, service evaluation, follow-up, implementation 
Implementation of patient-initiated reviews

\section{Abstract}

\section{Background}

The management of rheumatoid arthritis (RA) usually entails regular hospital reviews with a specialist often when the patient is well rather than during a period of exacerbation. An alternative approach where patients initiate appointments when they need them can improve patient satisfaction and resource use whilst being safe. This service evaluation reports a system wide implementation of a patient-initiated review appointment system called Direct Access (DA) for people with RA. The aim was to establish the impact on patient satisfaction of the new system versus usual care as well as evaluate the implementation processes.

\section{Method}

As all patients could not start on the new system at once, in order to manage the implementation, patients were randomly allocated to DA or to usual care. Instead of regular follow-up appointments, DA comprised an education session and access to a nurse-led telephone advice line where appointments could be accessed within two weeks. Usual care comprised routine follow-ups with the specialist. Data were collected on patient satisfaction, service use and outcomes of any contact to the advice line.

\section{Results}

Three hundred and eleven patients with RA were assessed as being suitable for DA. In terms of patient satisfaction, between-group differences were found in favour of DA for accessibility and convenience, ease of contacting the nurse and overall satisfaction with the service. Self-reported visits to the GP were also significantly lower. DA resulted in a greater number of telephone contacts (incidence rate ratio $=1.69 ; 95 \% \mathrm{Cl} 1.07$ to 2.68). Hospital costs of the two different service models were similar. Mean waiting time for an appointment was 10.8 days

\section{Conclusion}

This service evaluation found that DA could be implemented and demonstrated patient benefit in a real world setting. Further research establishing the broader cost-consequences across the whole patient pathway would add to our findings. 
Implementation of patient-initiated reviews

\section{Introduction}

Rheumatoid arthritis (RA) is a long-term condition that primarily affects the synovial joints causing unpredictable episodes of joint pain, stiffness and swelling. There are approximately 580,000 people with RA in the United Kingdom (UK), with a global prevalence of $0.24 \%$ (1). It has been estimated that the overall cost of RA to the UK economy is $f 8$ billion per year due to loss of productivity (2). People with RA often have lifelong symptoms that fluctuate daily, intermittent disease flares, and an unpredictable long-term outcome. This variation in experience makes it important for patients to develop the skills to manage their own condition and its consequences on their lives (3).

International recommendations state that people with stable RA should be reviewed every three to six months (4). Since patients are often seen on a date determined some time in advance, and review may occur when the patient is well rather than during periods of exacerbation (3), this traditional system of review may lead to a mismatch between patient need and clinical input. Although NICE recommends a minimum annual review, with access to additional specialist support for flares the burden of routine appointments can make it difficult to accommodate urgent care needs (5). A previous study into the content of routine reviews of patients with RA reported $42 \%$ were thought by a rheumatologist to be unnecessary (6). These findings suggest, first, that considerable hospital out-patient time and resources are wasted and, second, that the systems of review may be unresponsive at the times when patients need them most.

There is evidence to support an alternative model of RA management. A randomised controlled trial (RCT) compared standard rheumatologist-initiated review at three- to six-month intervals with patient-initiated review alone (7). The authors found that patient-initiated reviews maintained the patient's physical and psychological status but reduced unnecessary medical reviews by at least a third, thus making more efficient use of finite resources. The trial data also indicated that patients and primary-care physicians had more confidence and satisfaction in the new system compared with the standard model of routine follow-up. Two recent systematic reviews $(8,9)$ of patient-initiated 
Implementation of patient-initiated reviews

clinics for those with long-term conditions reported this was a safe approach, with some health service resource savings, and greater patient and clinician satisfaction.

This paper reports the outcomes from a project intended to implement a patient-initiated review system for RA at Plymouth Hospitals NHS Trust (PHNT) in the UK. Prior to the start of the project the PHNT had a clinician-driven follow-up system for people with RA - one which was overwhelmed and subject to delays. This study aimed to (a) establish the impact of a patient-initiated review system, called Direct Access (DA) for people with RA compared with usual follow-up on patient satisfaction and service use, and (b) to evaluate the implementation processes.

\section{Methods}

Study design

A prospective service evaluation, comparing DA with usual care was undertaken at PHNT from February 2012 to October 2013.

\section{Population}

People with RA aged 18 years or over, who had had RA for more than two years, and who were able to initiate telephone contact if needed were deemed eligible. We excluded people if they had no access to a telephone or were thought unable themselves or through a relative or friend to initiate telephone contact via the advice line when their condition required clinical review.

\section{Allocation and recruitment}

Three rheumatologists identified potentially eligible patients. Patients attending routine clinical review were then confirmed for suitability for the new system and were randomised to either DA or to usual care. We chose to randomise for two reasons; firstly due to the finite capacity of the education sessions (see below) meaning that all patients would not be able to enrol in DA at once, and secondly, it enabled the opportunity to undertake a prospective, comparative evaluation of DA 
Implementation of patient-initiated reviews

with the traditional clinician-led follow up model. The randomisation schedule was generated independently of the clinical and administrative team using computer generated random numbers. Any patients that declined to participate in the new system or did not attend an education session continued to receive their usual clinical care and regular reviews and were not included in the evaluation. As this was a service evaluation, ethical approval was not required and patients did not have to provide informed consent to take part. This was confirmed by the National Research Ethics Service South West (Bristol).

Interventions

Direct Access

Those allocated to the DA review system were sent an initial information sheet in the post by the administrative team explaining the system and offered a choice of dates to attend a patient education session. The patient education sessions were staggered over nine months due to limited departmental resources and were led by a rheumatology specialist nurse and involved groups of eight people with RA. The sessions focused on issues such as the operation of the new system, what patients can expect, when and how to call the advice line, when and how to ask for appointments. Patients were also able to raise any other queries they had regarding the system (3). Patients allocated to DA were not offered routine clinical reviews; their family doctor was informed of this and sent guidelines about how to manage problems commonly experienced by people with established RA. Patients or their family doctor could arrange prompt clinical advice and a review in clinic where required by calling a rheumatology nurse-led advice line where appointments could be accessed within two working weeks.

\section{$\underline{\text { Usual Care }}$}

Those people allocated to the control arm of the study continued to receive usual care in the form of planned appointments at regular intervals for a year before transferring to the new system. 
Implementation of patient-initiated reviews

Patients allocated to DA or usual care who had not contacted the advice line or been seen by either the consultant or nurse after a year were contacted for a clinical review with the rheumatology nurse specialist. This acted as a safety net (9).

\section{Data collection}

The primary outcome was patient satisfaction at twelve months, with secondary outcomes being service use and associated costs. We chose patient satisfaction as a surrogate measure of service quality. Process measures were also collected. Baseline demographic data were collected (age, gender, disease duration). We planned to collect the following clinical data at baseline and twelve months: total and swollen joint counts, early morning stiffness, pain in previous 24 hours, disease duration, disease activity score (DAS-CRP), Health Assessment questionnaire, patient and clinician global opinion of disease activity, C-reactive protein, plasma viscosity, haemoglobin, rheumatoid factor and rheumatoid factor titre (10). However, it quickly became clear that this was not going to be feasible within current clinical resources and was therefore abandoned.

\section{Patient satisfaction}

Patient satisfaction data were collected twelve months following randomisation using the Shortform Patient Satisfaction Questionnaire (PSQ-18) (11). The PSQ-18 comprises seven different subscales: general satisfaction, technical quality, interpersonal manner, communication, financial aspects, time spent with doctor, accessibility and convenience. Individual items were recorded on a five point Likert scale with ' 1 ' indicating strong agreement with statement and ' 5 ' indicating strong disagreement. Scoring on the sub-scales was calculated so that higher scores indicated satisfaction with medical care. After scoring, the median (interquartile range-IQR) scores of items within the same subscale were calculated. Five additional questions specifically relating to the local service satisfaction were also included: (a) ease of access to appointment with consultant, (b) ease of contacting specialist nurse by telephone, (c) satisfaction with telephone support, (d) satisfaction 
Implementation of patient-initiated reviews

with frequency of reviews, (e) satisfaction with own involvement in deciding frequency of reviews. The questions were scored using the same Likert scale that was used for the PSQ-18. The responses to the additional five questions were dichotomised and reported as a percentage. This was undertaken by grouping together (i) the two categories that indicated a favourable situation (i.e. satisfaction) and (ii) the three categories that indicated dissatisfaction or indifference. For the positively-worded questions this meant combining the "strongly agree" and "agree" categories but for one negatively-worded question it meant combining the "strongly disagree" and "disagree" categories. Patients were also asked to indicate their satisfaction with overall rheumatology care on a scale from 0 (completely unsatisfied) to 100 (completely satisfied). Satisfaction data were collected by a rheumatology healthcare assistant over the telephone.

\section{Service use}

Hospital patient administration systems were used to collect data on number of contacts to the nurse-led telephone advice line and face-to-face contacts with the rheumatologist or nurse specialist over the twelve-month period following randomisation. Patients were asked to report the number of times they saw their general practitioner regarding their RA during the previous year.

\section{Process measures}

Process measures for DA included the outcome of phoning the advice line and whether a face-toface appointment was needed and, if so, who this was with (rheumatologist or nurse specialist). The duration between phoning the advice line and having a face-to-face appointment was also recorded. The review systems were monitored to ascertain whether they were undertaken as planned.

Sample size

The assumptions underlying the sample size calculation were derived from a published RCT (12). The current evaluation required 190 subjects followed up for a period of 12 months in each of the DA 
Implementation of patient-initiated reviews

and usual care groups in order to detect a decrease in the number of consultations from 2.5 per year to 2 per year with $90 \%$ power at the $5 \%$ level of significance level.

\section{Analysis}

A CONSORT flow chart was used to describe the flow of patients in the study (Figure 1). Descriptive statistics were used to report the characteristics of patients allocated to each study arm. The Mann Whitney test was used to compare quantitative outcomes between the DA and usual care groups and the Chi-squared test to compare binary outcomes. The median (interquartile range - IQR) was calculated for number of GP visits and overall satisfaction.

The occurrence of telephone and face-to-face contacts was compared between the DA and usual care groups using Poisson regression to analyse the number of contacts over 12 months. The rate of contact (based on the total number of contacts and person-years of patient follow-up) was reported for each group together with the incidence rate ratio (IRR), 95\% confidence interval and $p$-value.

For those allocated to the intervention, the mean (SD) waiting time from telephone contact to faceto-face appointment was calculated, and the proportion of people being seen within the target of two working weeks.

Costs, from a secondary care perspective, of delivering DA and usual care were estimated on a per participant basis and reported descriptively. During the 12 month follow-up period, individual level data were identified from hospital records on: i) the number of telephone contacts with a rheumatology nurse; ii) the number of face-to-face contacts with a rheumatology nurse and; iii) the number of face-to-face contacts with a consultant rheumatologist. The length of time of telephone calls and face-to-face contacts was estimated via discussion with clinical staff. In addition, the resource requirements for the education sessions were also determined by discussion with clinical 
Implementation of patient-initiated reviews

staff. Nationally-recognised unit costs (13), as set-out in Table 1, were applied to the estimated service use.

**Table $1 * *$

Results

Figure 1 shows the patient flow through the study. One hundred and fifty six patients were allocated to DA with 155 allocated to usual care. Four patients that had been allocated to DA were assessed as being clinically unsuitable to continue during follow-up resulting in a need to resume regular clinical reviews. The mean age (SD) of DA and usual care participants was 65.4 (11.7) and 63.1 (13.1) respectively. One hundred and four DA patients were female (75.4\%) as were 118 (76.6\%) of usual care participants.

\section{**Figure $1 * *$}

Patient satisfaction questionnaire data were available from 80/138 (58\%) of intervention arm patients and from 98/155 (64\%) of control arm participants at one year follow-up (Table 2). Between-group differences were found in favour of DA for accessibility and convenience, ease of contacting specialist nurse and overall satisfaction with the service. Interpersonal manner was significantly higher for usual care. There were no between-group differences in other aspects of patient satisfaction. In terms of service use (Table 3), self-reported visits to GP were significantly lower for DA participants when compared with usual care (median 0 and 1 , respectively; $p=0.03$ ). There was evidence of an increase in telephone contacts by those using DA (IRR $=1.69 ; 95 \% \mathrm{Cl} 1.07$, 2.68). There was no significant difference in the number of hospital based face-to-face consultations $($ IRR $=0.92 ; 95 \% \mathrm{Cl} 0.70,1.20)$ although a smaller proportion of DA consultations were with a rheumatologist when compared with usual care consultations (59\% vs. $79 \%$ ).

**Table $2^{* *}$ 
Implementation of patient-initiated reviews

**Table $3^{* *}$

After contacting the advice line $44 / 46$ (96\%) patients required a face-to-face appointment. Of those intervention group patients needing a face-to-face appointment 91\% (40/44) were seen within 14 calendar days (10 working days). The mean (SD) waiting time from telephone contact to face-to-face appointment was 10.8 (10.3) days, ranging from being seen the same day to waiting for 70 days. Median (IQR) waiting time was 10 (7 to 13$)$ days.

The resource use and costs of the two models of service delivery are presented in Table 4. This indicates the different patterns of resource use, and shows a greater cost of telephone contacts and face-to-face appointments with the rheumatology nurse in the intervention group, and a greater cost of face-to-face appointment with the rheumatology consultant in the control group. When the cost of the educational session was added, the total cost of service delivery by both models was broadly similar.

**Table $4 * *$

\section{Discussion}

This study aimed to evaluate the implementation of a patient-initiated review system, known as Direct Access, for people with rheumatoid arthritis. For most subscales of the PSQ-18 there were no statistical significant differences between DA and usual care unlike the findings of from Whear and colleagues (8). The different findings may relate to the varied ways in which satisfaction is conceptualised and measured. There was, however, a significant difference in favour of DA with relation to PSQ-18 sub-scale for accessibility and convenience, ease of contacting the nurse specialist and overall satisfaction. The issue of accessibility is supported by the findings of the nested qualitative study that took place in parallel to this evaluation (14) that found that being able to access the service when they needed, rather than when they were well was a better use of patients and staff time. Being in control of accessing care when needed is important to people with long term 
Implementation of patient-initiated reviews

conditions (15) and recent discussions with our local population with RA and other long-term conditions highlighted that they want more accessible and responsive services to meet their needs in a timely, effective and efficient way. We also found a difference, in terms of interpersonal manner, in favour of usual care. The reasons for this are unclear as it was the same clinical team seeing the patients.

No difference in terms of secondary care service use was found which is in contrast to Hewlett and colleagues who reported $44 \%$ fewer reviews with the consultant for those using a patient-initiated service (7). However, we reported that those who remained on the usual care pathway were seen, on average only 0.73 times per year; considerably lower than the 2.5 appointments per year that had been estimated based on previous research (7), whereas those using DA were seen on average only 0.6 times in a year. This reduction in usual care provision was due in part to reduced staffing and an increasing waiting list, and because NHS priorities are for seeing people for the initial appointment (16) with no targets around follow-up, thus rendering follow-up appointments less of a priority. We found no evidence of 'overuse' of DA, with the largest number of contacts from a single patient being three over the course of a year. This challenges the view that this type of appointment would need to be capped (17). An unexpected finding of our study was the significant reduction in patient-reported visits to the GP, as there had been concern amongst the clinicians that there could potentially be a shift towards an increased primary care burden. We found broadly similar costs for DA and usual care although these could be viewed as conservative as usual care participants had not received the number of anticipated appointments. There may well be potential cost savings by implementing a patient-initiated review system in services that currently see patients more frequently than the 0.73 times a year we observed. The main cost drivers of the different models are set out here, but we did not take into account potential broader costs, such as contacts with other healthcare professionals e.g., physiotherapists, or other costs such as prescribing, hospital admissions or community-based health or social care provision. The collection of such data may 
Implementation of patient-initiated reviews

have given added value to the findings but its collection was unsustainable within existing overstretched clinical services.

Only $9 \%$ of patients were not seen within two weeks of contacting the advice line. The reasons for this include the patient preferring to delay and see their usual specialist, and public holidays resulting in fewer clinic slots available those weeks. Advance clinic planning is essential for those times where additional capacity is needed to avoid the system breaking down (18).

A further difference observed in the different service delivery models was the type of follow-up with a greater proportion of people requiring specialist review being seen by a nurse rather than a rheumatologist. The increased autonomy and clinical skills, such as independent prescribing, of the nursing profession in the UK has seen a shift in the skill mix of service provision with non-medical health professionals undertaking roles that had traditionally been undertaken by physicians (19).

A number of limitations were noted that related to methods, data collection and intervention delivery. The first limitation was the failure to recruit the sample size within the time frame of the evaluation. This was due to the practical challenge of identifying potentially suitable participants during routine clinical practice rather than a lack of potential participants. Secondly, there is uncertainty whether the participants in the two study arms were similar at baseline in terms of clinical characteristics, due to extensive missing data. This is the challenge of undertaking a formal evaluation of clinical practice and service delivery without additional data collection resources. However, the two groups appear broadly similar in terms of gender and age and the nature of the randomisation process should potentially mitigate this limitation. Future evaluations should utilise other approaches to maximise patient-reported data collection, use routine electronic disease assessment data, and consideration should be made to the quantity and type of outcome data collected. Fewer clinical and symptom measures and a focus on what outcomes are important to patients (20), such as quality of life, should be considered in any future evaluations (21). We are currently developing a toolkit for implementing DA with other patient groups and one key area is 


\section{Journal of Evaluation in Clinical Practice}

Implementation of patient-initiated reviews

selecting outcomes that are important to those patients that can be collected in a sustainable way as a routine part of the service. A third limitation was that low staffing levels impacted on the ability to review patients at the safety net appointment at twelve months. The safety net is important for those not able to recognise changes in disease state that may require clinical intervention such as those with cognitive impairment or depression. However, DA education should, where possible, ensure that a family member or friend attend the education session and act as an advocate so they know how and when to access the service. For some though this may not be possible and therefore DA may not be appropriate as significant deterioration may occur and go unreported. Despite the limitations, we undertook an evaluation within a real world setting, experiencing the challenges of undertaking an evaluation without the traditional structures of a formal research study. We were able to undertake a prospective, comparative study using randomisation which has provided valuable information for future the implementation of patient-initiated reviews.

After a clinical department review there is now a face-to-face review at eighteen months alongside a letter sent to patients that have not been seen for a year. This, however, is contrary to the annual review recommended by NICE (5) but is a pragmatic response to freeing clinical capacity whilst maintaining reasonable contact with an individual patient. Notably, there is evidence from the work in Bristol that patient-initiated follow-up is not detrimental to clinical or psychological outcomes when compared with clinician-initiated care, even after six years (12).

\section{Conclusion}

This evaluation of implementing research into practice in terms of a patient-initiated review service for people with rheumatoid arthritis was found to have benefits over a traditional regular review in terms of convenience and accessibility, ease of contacting a nurse specialist and reduced GP appointments. In terms of cost, the service was similar to a routine follow-up service. However, further evaluation including broader cost-consequences analysis across the whole patient pathway would add to the current findings as would a longer follow-up. This evaluation is contributing to the 
Implementation of patient-initiated reviews

development of a toolkit for supporting the implementation of patient-initiated reviews in other specialities and patient groups.

\section{Acknowledgements}

We would like to thank the Rheumatology team and patients from Plymouth Hospitals NHS Trust. This research was funded by the National Institute for Health Research (NIHR) Collaboration for Leadership in Applied Health Research and Care South West Peninsula. The views expressed are those of the author(s) and not necessarily those of the NHS, the NIHR or the Department of Health. 
Implementation of patient-initiated reviews

\section{References}

1. Cross $M$, Smith $E$, Hoy D, et al. The global burden of rheumatoid arthritis: estimates from the global burden of disease 2010 study. Ann Rheum Dis. 2014 Jul;73(7):1316-22.

2. National Rheumatoid Arthritis Society. The economic burden of rheumatoid arthritis. Maidenhead, UK2010.

3. Pope D, Tipler S, Kirwan J, Hewlett S. Implementing a patient-led service for chronic conditions. Nurs Times. 2005;101:28 - 31.

4. Smolen JS, Aletaha D, Bijlsma JW, et al. Treating rheumatoid arthritis to target: recommendations of an international task force. Ann Rheum Dis. 2010 Apr;69(4):631-7.

5. National Institute for Health and Care Excellence. Quality Standard (QS33): Rheumatoid arthritis Manchester: NICE,2013.

6. Hehir M, Hewlett S, Mitchell K, Kirwan J, Memel D, Pollock J, Bennett B. What happens in rheumatoid arthritis (RA) out-patient clinics? Rheumatology. 2001;40(S1):S140.

7. Hewlett S, Mitchell K, Haynes J, Paine T, Korendowych E, Kirwan J. Patient-initiated hospital follow-up for rheumatoid arthritis. Rheumatology. 2000;39:990 - 7.

8. Whear R, Abdul-Rahman A-K, Thompson-Coon J, Boddy K, Perry M, Stein K. Patient initiated clinics for patients with chronic or recurrent conditions managed in secondary care: a systematic review of patient reported outcomes and patient and clinician satisfaction. BMC Health Services Research. 2013;13(1):501.

9. Whear R, Abdul-Rahman AK, Boddy K, Thompson-Coon J, Perry M, Stein K. The clinical effectiveness of patient initiated clinics for patients with chronic or recurrent conditions managed in secondary care: a systematic review. PLoS One. 2013;8(10):e74774.

10. Paudyal P, Perry M, Child S, Gericke C. Evaluation of a patient-initiated review system in rheumatoid arthritis: an implementation trial protocol. BMC Musculoskeletal Disorders. 2012;13(1):120.

11. Marshall G, Hays R. The patient satisfaction questionnaire short form (PSQ-18). Santa Monica: RAND Corporation1994.

12. Hewlett S, Kirwan J, Pollock J, Mitchell K, Hehir M, Blair P, Memel D, Perry M. Hospital follow up in chronic disease: A randomised controlled trial of routine out-patient follow-up versus patientinitiated direct access in rheumatoid arthritis, extended to 6 years. BMJ. 2005;330(7484):171 - 5.

13. Curtis L. Unit costs of health and social care. Canterbury: University of Kent2013.

14. Child S, Goodwin VA, Perry MG, Gericke CA, Byng R. Implementing a patient-initiated review system in rheumatoid arthritis: a qualitative evaluation. BMC Health Serv Res. 2015;15:157.

15. Foot C, Gilburt H, Dunn P, Jabbal J, Seale B, Goodrich J, Buck D, Taylor J. People in control of their own health and care: the state of involvement London: The King's Fund2014.

16. Department of Health. 2014/15 Choice Framework. In: NHS and Finance Directorate, editor.: Department of Health; 2014.

17. Bukhari $M$, Bamji AN, Deighton $C$. Is it ever appropriate to discharge patients with rheumatoid arthritis? Rheumatology. 2007 November 1, 2007;46(11):1631-3.

18. Gupta D, Denton B. Appointment sceduling in health care: challenges and opportunities. IIE Transactions. 2008;40:800-18.

19. Kinley H, Czoski-Murray C, George S, et al. Extended scope of nursing practice: a multicentre randomised controlled trial of appropriately trained nurses and pre-registration house officers in pre-operative assessment in elective general surgery. Health Technol Assess. 2001;5(20):1-87.

20. Sanderson T, Morris M, Calnan M, Richards P, Hewlett S, Ph DsfARC. What outcomes from pharmacological treatments are important to people with rheumatoid arthritis? Creating the basis of a patient core set. Arthritis care \& research. 2010;62(5):640-6.

21. Deshpande PR, Rajan S, Sudeepthi BL, Abdul Nazir CP. Patient-reported outcomes: A new era in clinical research. Perspectives in Clinical Research. 2011 Oct-Dec;2(4):137-44. 
Implementation of patient-initiated reviews

Figure legends

Figure 1 Flow chart of participants though the evaluation 
Implementation of patient-initiated reviews

Table 1 Unit costs

\begin{tabular}{|c|c|c|c|}
\hline $\begin{array}{l}\text { Resource use } \\
\text { item }\end{array}$ & $\begin{array}{c}\text { Unit cost } \\
(f, 2012 / 2013)\end{array}$ & Source & Basis of estimate \\
\hline $\begin{array}{l}\text { Educational } \\
\text { session (per } \\
\text { participant) }\end{array}$ & f8.94 & $\begin{array}{c}\text { Personal Social } \\
\text { Services Research } \\
\text { Unit (PSSRU) }\end{array}$ & $\begin{array}{l}1 \text { hour group (8 participants) educational } \\
\text { session with a Band } 7 \text { nurse manager } \\
\text { (plus } 5 \text { hours development time) }\end{array}$ \\
\hline $\begin{array}{l}\text { Telephone } \\
\text { contact }\end{array}$ & £8.17 & PSSRU & $\begin{array}{c}10 \text { minute telephone conversation with } \\
\text { a Band } 6 \text { nurse team leader }\end{array}$ \\
\hline $\begin{array}{c}\text { Nurse } \\
\text { appointment }\end{array}$ & $f 16.33$ & PSSRU & $\begin{array}{c}20 \text { minute face to face appointment with } \\
\text { a Band } 6 \text { nurse team leader }\end{array}$ \\
\hline $\begin{array}{l}\text { Consultant } \\
\text { appointment }\end{array}$ & f46.33 & PSSRU & $\begin{array}{l}20 \text { minute face to face appointment with } \\
\text { a medical consultant }\end{array}$ \\
\hline
\end{tabular}


Implementation of patient-initiated reviews

Table 2 Patient satisfaction at one-year follow-up

\begin{tabular}{lccc}
\hline Outcome & Direct Access & Usual care & p-value \\
Median (IQR) & Median (IQR) \\
& $\mathrm{N}=80$ & $\mathrm{~N}=98$ & \\
\hline
\end{tabular}

Patient Satisfaction Questionnaire

(PSQ-18) Sub-scales

\begin{tabular}{|c|c|c|c|}
\hline General Satisfaction & $4(4$ to 4$)$ & $4(3$ to 4$)$ & 0.79 \\
\hline Technical Quality & $4(4$ to 4$)$ & 4 (3.5 to 4.25$)$ & 0.73 \\
\hline Interpersonal Manner & 4 (4 to 4$)$ & 4 (4 to 4.5$)$ & 0.01 \\
\hline Communication & $4(4$ to 4$)$ & 4 (4 to 4.5$)$ & 0.23 \\
\hline Financial Aspects & 4 (4 to 4.5$)$ & 4 (4 to 4.5$)$ & 0.11 \\
\hline Time Spent with Doctor & $4(4$ to 4$)$ & $4(3.5$ to 4.5$)$ & 0.38 \\
\hline Accessibility and Convenience & 3.75 (3.5 to 4$)$ & 3.5 (3 to 4$)$ & 0.02 \\
\hline \multicolumn{4}{|l|}{ Satisfaction with local service } \\
\hline $\begin{array}{l}\text { Found it easy to get appointment } \\
\qquad \text { with doctor, \% }\end{array}$ & $67 \%$ & $54 \%$ & 0.07 \\
\hline $\begin{array}{l}\text { Did not find it difficult to contact } \\
\text { specialist nurse, } \%\end{array}$ & $63 \%$ & $46 \%$ & 0.03 \\
\hline $\begin{array}{r}\text { Satisfied with telephone support } \\
\text { received, \% }\end{array}$ & $67 \%$ & $56 \%$ & 0.12 \\
\hline $\begin{array}{r}\text { Satisfied with how often reviewed, } \\
\%\end{array}$ & $72 \%$ & $66 \%$ & 0.38 \\
\hline $\begin{array}{l}\text { Satisfied with amount of personal } \\
\text { involvement in deciding frequency } \\
\text { of review, \% }\end{array}$ & $61 \%$ & $53 \%$ & 0.28 \\
\hline $\begin{array}{r}\text { Satisfied with service }(100 \mathrm{~mm} \text { visual } \\
\text { analogue scale) }\end{array}$ & 100 (80 to 100$)$ & 80 (70 to 100$)$ & 0.002 \\
\hline
\end{tabular}


Implementation of patient-initiated reviews

Table 3 Description of service use over a year

\begin{tabular}{lcc}
\hline & $\begin{array}{c}\text { Direct Access } \\
\mathbf{N}=\mathbf{1 3 8}\end{array}$ & $\begin{array}{c}\text { Usual care } \\
\mathbf{N}=\mathbf{1 5 2}\end{array}$ \\
\hline $\begin{array}{l}\text { Number of GP visits, median } \\
(\text { IQR) }\end{array}$ & $0(0$ to 2) & 1 (0 to 3) \\
\hline $\begin{array}{l}\text { Number of patients/advocates } \\
\text { contacting nurse advice line (\%) }\end{array}$ & $36(26 \%)$ & $30(20 \%)$ \\
\hline $\begin{array}{l}\text { Total number of phone } \\
\text { contacts to nurse advice line }\end{array}$ & 46 & 30 \\
\hline $\begin{array}{l}\text { Number of patients having } \\
\text { face-to-face consultation (\%) }\end{array}$ & $78(47 \%)$ & $102(67 \%)$ \\
\hline $\begin{array}{l}\text { Total number of face-to-face } \\
\text { consultations }\end{array}$ & 94 & 89 \\
\hline \multicolumn{1}{c}{- Doctor } & 55 & 24 \\
\hline
\end{tabular}


Implementation of patient-initiated reviews

Table 4 Description of resource use and costs of delivering Direct Access and Usual Care

\begin{tabular}{|c|c|c|c|c|}
\hline & \multicolumn{2}{|c|}{$\begin{array}{c}\text { Mean (SD) number of contacts } \\
\text { per participant }\end{array}$} & \multicolumn{2}{|c|}{$\begin{array}{c}\text { Mean (SD) cost }(\mathbf{f}) \\
\text { per participant }\end{array}$} \\
\hline & $D A$ & Usual care & $D A$ & Usual care \\
\hline & $N=138$ & $N=152$ & $N=138$ & $N=152$ \\
\hline $\begin{array}{l}\text { Educational } \\
\text { session }\end{array}$ & 1 & - & 8.94 & \\
\hline $\begin{array}{l}\text { Telephone } \\
\text { contacts }\end{array}$ & $0.33(0.63)$ & $0.20(0.40)$ & $2.72(5.16)$ & $1.61(3.26)$ \\
\hline $\begin{array}{l}\text { Face-to-face nurse } \\
\text { appointments }\end{array}$ & $0.28(0.45)$ & $0.15(0.38)$ & $4.61(7.37)$ & $2.57(6.25)$ \\
\hline $\begin{array}{l}\text { Face-to-face } \\
\text { consultant } \\
\text { appointments }\end{array}$ & $0.40(0.68)$ & $0.61(0.55)$ & $18.45(31.42)$ & $28.02(25.64)$ \\
\hline Total cost & & & $34.72(34.51)$ & 32.21 (22.15) \\
\hline
\end{tabular}


Implementation of patient-initiated reviews

Figure 1 Flow chart of participants though the evaluation

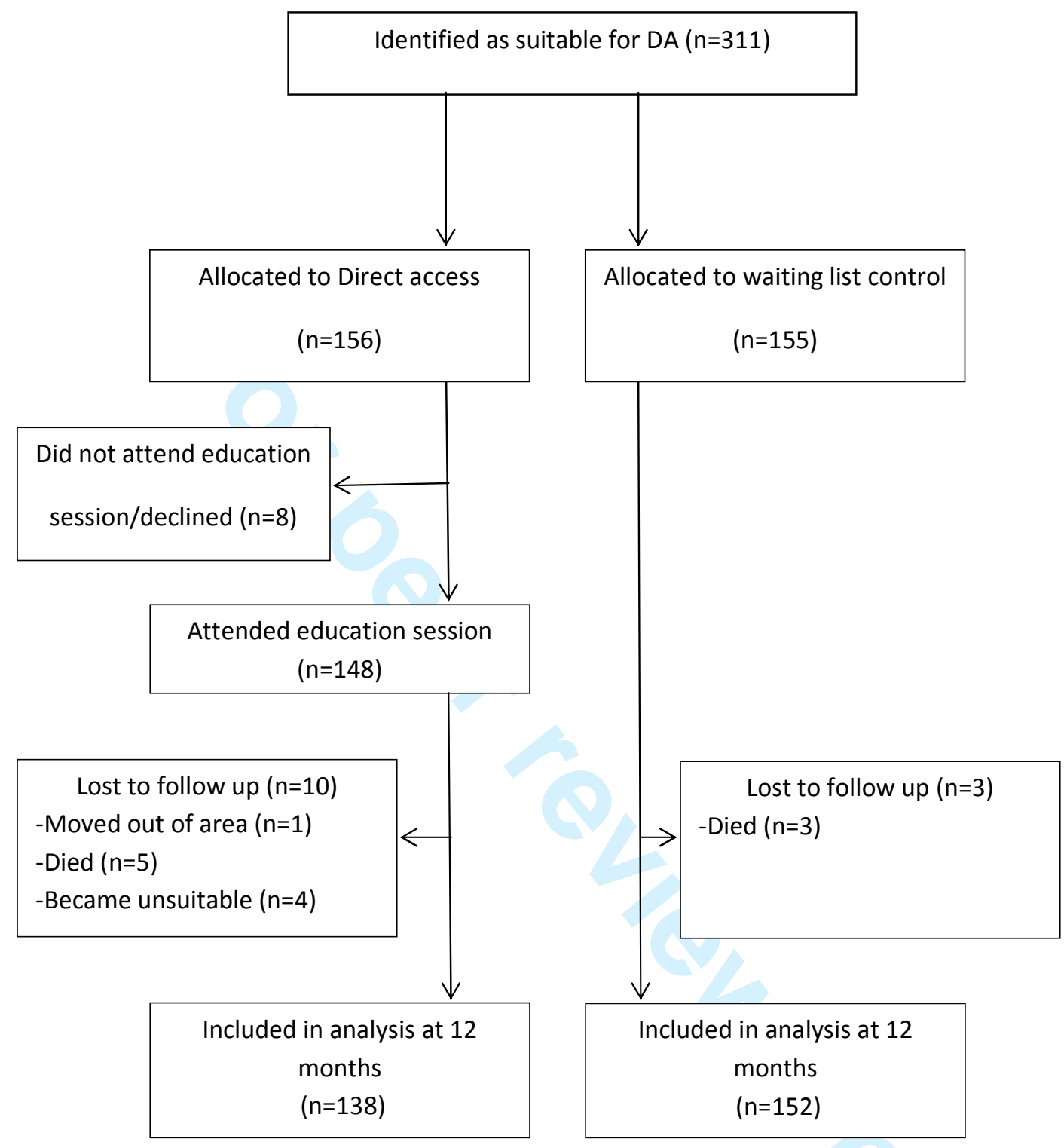

